Fuzziness in Information Systems 
Miroslav Hudec

\section{Fuzziness in Information Systems}

How to Deal with Crisp and Fuzzy Data in Selection, Classification, and Summarization

Springer 


\author{
Miroslav Hudec \\ Faculty of Economic Informatics \\ University of Economics in Bratislava \\ Bratislava \\ Slovakia
}

ISBN 978-3-319-42516-0

ISBN 978-3-319-42518-4 (eBook)

DOI 10.1007/978-3-319-42518-4

Library of Congress Control Number: 2016948117

(C) Springer International Publishing Switzerland 2016

This work is subject to copyright. All rights are reserved by the Publisher, whether the whole or part of the material is concerned, specifically the rights of translation, reprinting, reuse of illustrations, recitation, broadcasting, reproduction on microfilms or in any other physical way, and transmission or information storage and retrieval, electronic adaptation, computer software, or by similar or dissimilar methodology now known or hereafter developed.

The use of general descriptive names, registered names, trademarks, service marks, etc. in this publication does not imply, even in the absence of a specific statement, that such names are exempt from the relevant protective laws and regulations and therefore free for general use.

The publisher, the authors and the editors are safe to assume that the advice and information in this book are believed to be true and accurate at the date of publication. Neither the publisher nor the authors or the editors give a warranty, express or implied, with respect to the material contained herein or for any errors or omissions that may have been made.

Printed on acid-free paper

This Springer imprint is published by Springer Nature

The registered company is Springer International Publishing AG Switzerland 
I dedicate this book to Martina. I would like to skip writing reasons, because this part should be shorter than a book chapter. 


\section{Foreword}

It is a pleasure to write this foreword because this book is an important contribution to the literature on applications of fuzzy models. There are many books dealing with fuzzy sets in a general way but this work is an essential contribution to the description of fuzziness in information systems.

Usually in statistical information systems data are stored as numbers which pretend a precision which is not justified, because real data are frequently not available as precise numbers but they are more or less non-precise. This imprecision is different from errors and it is best modelled by the so-called fuzzy numbers, which are special fuzzy subsets of the set of real numbers. To describe fuzziness in quantitative mathematical terms, is an important innovation in science and management.

When Karl Menger introduced the generalization of classical sets in the year 1951 by generalizing the indicator function of classical sets, this was a theoretical concept and it took many years until practical applications of these generalized sets came up. An important step was the paper by Lotfi Zadeh in 1965 when he introduced the name fuzzy set and defined generalized set operations based on the defining functions of fuzzy sets. These defining functions were called membership functions and in the last decades of the twentieth century enormous research activities developed the theory and applications of fuzzy sets. The concept of fuzziness was extended to generalize logics, and the calculus of fuzzy logics was created. In the meantime fuzzy concepts are applied in many scientific fields, for example in civil engineering for risk analysis of structures, in medical science for diagnostic systems, in measurement science to describe results of precision measurement, in statistics for the description and analysis of real data, in Bayesian analysis to model fuzzy a priori information, in information science to describe fuzzy information and to formulate fuzzy questions.

Chapter 1 of the book gives an introduction to fuzzy sets and fuzzy logic, linguistic variables, fuzzy quantifiers, and related references.

Chapter 2, Fuzzy Queries, considers the way from crisp to fuzzy queries, the construction of fuzzy sets for flexible conditions, the conversion of fuzzy conditions 
to SQL ones, the calculation of matching degrees, empty and overabundant answers, and some issues related to practical realization.

Chapter 3, Linguistic Summaries, explains the benefits of linguistic summarization (LS), the basic structure of LS, relative quantifiers, quality measures of LS, applicability of LS, and building summaries.

Chapter 4, Fuzzy Inference, is devoted to fuzzy models for control systems. After introducing fuzzy inference engines, the chapter presents a section on fuzzy classification, and concludes with remarks to applications.

The next chapter, Fuzzy Data in Relational Databases, is central to the book. It starts with the classical relational databases, has a section on fuzziness in the real world, explains fuzzy databases and their basic model, fuzzy data in traditional relational databases, aggregation functions in queries, and linguistic summaries on fuzzy data.

Chapter 6, Perspectives, Synergies and Conclusion, briefly explains the relationship between fuzzy inference and fuzzy databases and linguistic summaries as well as fuzzy queries.

The references at the end of each chapter are helpful for further reading.

Appendixes, Illustrative Interfaces and Applications for Fuzzy Queries and Illustrative Interfaces and Applications for Linguistic Summaries, and an Index are helpful for the reader.

This book explains important applications of fuzzy logic in information systems. Congratulations to the author for this valuable and up-to-date contribution to information science.

Wien

Reinhard Viertl

April 2016 


\section{Preface}

The increasing use of information systems by governmental agencies and businesses has created mountains of data that contain potentially valuable knowledge. Admittedly, these data do constitute "golden mines" which should be swiftly and efficiently processed and interpreted to be useful. Users (e.g. decision-makers) would like to efficiently reveal relevant data. Moreover, users are often not interested in large sheets of figures, but in knowledge that is usually overshadowed by large amount of data.

People can relatively easily answer imprecise questions like, is it true that most of tall persons in the room wear blue or green shirts? Different hues of these colours as well as the meaning of the vague term tall people are not limitations for solving this task. However, if we want to know, which of these two sentences: most of young commuters commute short distances; most of medium aged commuters commute short distances better explains the commuting behaviour, then we have to adapt this query to mine the truth value form the data. The same holds for querying cheap hotel with good references and if possible near to the city centre and common-sense reasoning: if customer buys products very often, then provide high discount.

The initial research in the theory of fuzzy sets and fuzzy logic was motivated by the perception that traditional computing techniques are not effective in dealing with problems, in which vagueness, imprecision and subjectivity are immanent, and therefore should not be neglected. These types of uncertainty are commonly called fuzziness.

According to Prof. Zadeh, four principal rationales for handling fuzziness exist. Two of them, which are relevant for this book, are: "don't need rationale" and "don't know rationale". In the former, the tolerance for imprecision is in accord with the remarkable human capability to solve variety of tasks without precise calculations. For example, summarizing data by short questions of natural language; creating queries with flexible conditions and approximate inference. In the latter, the values of attributes are not known with sufficient precision to justify the use of traditional databases for storing these data. Many data cannot be adequately expressed as precise numbers or as one linguistic term, due to non-sharp 
boundaries of observations, tendency of people to estimate or guess answers in surveys and tolerance intervals of measurement instruments. Therefore, the data are often vague and include both quantitative and qualitative elements. Storing these data as crisp values might cause loss of valuable information.

Keeping in mind the aforementioned facts, fuzzy queries, fuzzy inference processes, linguistic summaries and managing fuzzy data in information systems could be the option. We have chosen these areas, because businesses of all sizes and governmental agencies cope with them in their work. The motivation for this book has arisen from the author's experience in teaching courses of fuzzy logic for business informatics and database design and in research and development of information systems and data mining applications mainly for the official statistics purposes. Furthermore, many small- and medium-sized enterprises cannot afford sophisticated tools or experts for information systems and data mining, even though they are aware of limitation of sharp boundaries in data analyses. Many tasks can be solved in a classical way, but their complexity becomes high. The complexity of the problem can be reduced by including the intensity of the examined property. This permits us to discern elements with the same property, based on the intensity matching it.

Roughly, the intent of the book could be depicted in Fig. 1. The usual scenario is that user wants to retrieve data or summarized information from a database. Furthermore, user might be interested to classify data. Often user is not aware of the nature of collected data or cannot determine sharp criteria. In addition, all data including vague ones are usually stored as crisp values.

In the book we examine these approaches theoretically as well as on the municipal statistics data. The latter is illustrated in appendixes. These data are suitable source, due to larger number of municipalities, which are often very similar in several attributes. Second reason is that some of attributes are fuzzy in their nature, but are limited to crisp values.

We should not expect that domain experts are familiar with the fuzzy logic theory. Therefore, the book demonstrates developing user-friendly interfaces to allow users exploring advantages of fuzzy logic in their tasks. Furthermore,

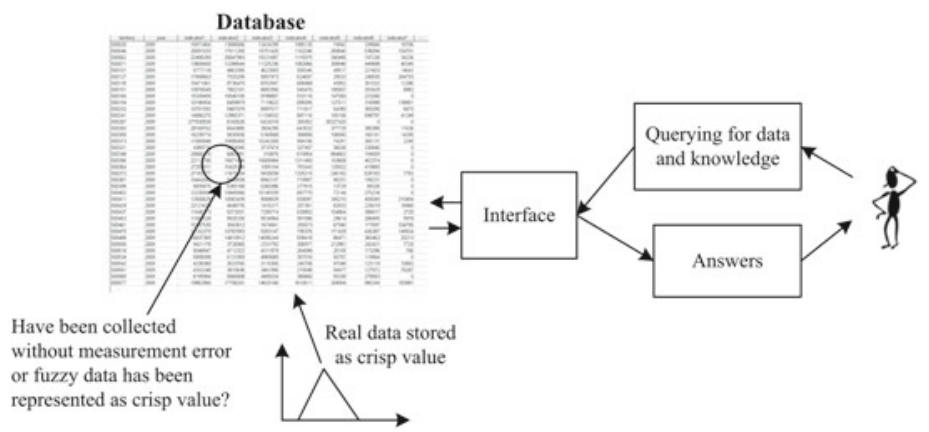

Fig. 1 Intent of the book 
companies usually keep data in relational databases. We should keep this in mind during adapting database to cope with the fuzzy data.

The main target audience of the book are students, researchers and practitioners working in fields of data analysis, database design and business intelligence. This book does not go deeply into the foundation and the mathematical theory of fuzzy logic and relational algebra (e.g. theorems' proofs). Hence, intermediate knowledge of fuzzy logic and relational databases is recommended.

The book is divided into six chapters in the following way. Chapter 1 is focused on the theory of fuzzy sets and fuzzy logic to a level, which is advisable to know in order to proceed to next chapters. Readers skilled in fuzzy logic theory can skip this chapter.

Chapter 2 is devoted to flexible queries. The following aspects of flexible queries are examined: constructing fuzzy sets for query conditions; aggregation operators for commutative and non-commutative conditions with and without priorities; dealing with empty and overabundant answer problems and issues related to practical realizations.

Chapter 3 is dedicated to linguistic summaries. We start with the basic linguistic summary and build more complex ones. To meet this goal, selecting appropriate aggregations, implications for preferences and issues related to construction of membership functions are examined. Quality measures of created summaries are also considered. Finally, several possible applicabilities are discussed.

Chapter 4 presents fuzzy logic control architecture adjusted to the aims of business and governmental agencies. It shows fuzzy rules, construction of fuzzy sets and procedures for solving inference tasks by generalized modus ponens. In the first part we explain reasoning procedures. In the second part fuzzy expert systems are discussed. In the last part classification by IF-THEN rules is examined.

Chapter 5 covers fuzzification of classical relational databases. We briefly review classical relational databases and fuzzy database models. The emphasis is on storing fuzzy data in classical relational databases in a way that existing data and normal forms are not affected. Furthermore, practical aspects of user-friendly interfaces for storing, updating, querying and summarizing are examined.

Chapter 6 shortly discusses possible integration of fuzzy queries, summarization and inference related to crisp and fuzzy databases. Use of these approaches in a complementary, rather than competitive way, can support variety of tasks.

Finally, we suppose that the book will provoke at least some interest to continue research and also will be of support for developing tailored applications communicating with users by easy-to-use interfaces. Maybe the next generations of relational database management systems and applications will include many fuzzy characteristics and users will enjoy easy-to-use interfaces for fuzzy queries, fuzzy inferences, fuzzy summarization, fuzzy recommending and so on, without the necessity of knowing mathematics of fuzzy logic. We hope that the book will contribute to this field with a membership degree greater than 0.25 . 


\section{Acknowledgements}

Advice and suggestions touching theoretical as well as practical implications related to my work were valuable source for this research. In this direction, I express my deep appreciation to Tomáš Bacigál, Simona Balbi, Mojca Bavdaž, Piet Daas, Boris Delibašić, Erich Peter Klement, Gabriela Kristová, Andreas Meier, Radko Mesiar, Dušan Praženka, Dragan Radojević, Agnieszka Stawinoga, František Sudzina, Matthias Templ, Valentin Todorov, Vanessa Torres van Grinsven, Steven Vale, Enrique Herrera-Viedma, Reinhard Viertl, Miljan Vučetić and Mirko Vujošević.

I also address my gratitude to the authors of publications listed in the references. Their contributions were inspiration for my work and are reflected in the book.

Concerning data required for experiments I thank Statistical Office of the Slovak Republic for providing the data. I am grateful to Eleonora Vallová for proofreading and editing my manuscript.

I am also grateful to Springer for offering me opportunity to publish my humble view and opinion about the fuzziness in their publishing house and especially Ralf Gerstner for the cooperation during the whole process.

My final thanks go to the heads of the Faculty of Economic Informatics and especially Department of Applied Informatics for providing me with space for research which enabled me to spend long hours in researching and writing the book. 


\section{Contents}

1 Fuzzy Set and Fuzzy Logic Theory in Brief $\ldots \ldots \ldots \ldots \ldots \ldots$. 1

1.1 From Crispness to Fuzziness $\ldots \ldots \ldots \ldots \ldots \ldots \ldots \ldots \ldots \ldots$

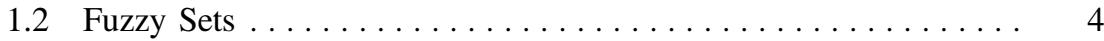

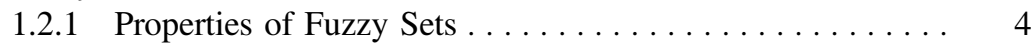

1.2.2 Types of Fuzzy Sets (Membership Functions). . . . . . 7

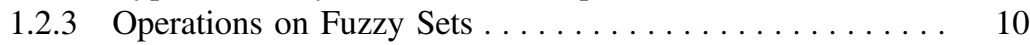

1.2.4 Fuzzy Numbers and Fuzzy Arithmetic . . . . . . . . . . 13

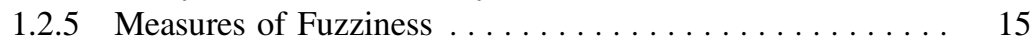

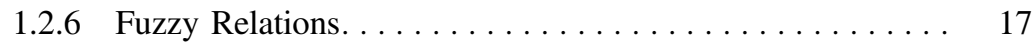

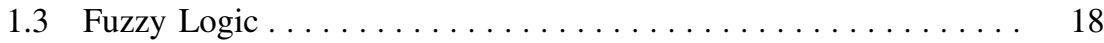

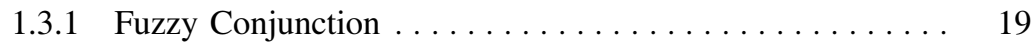

1.3 .2 Fuzzy Negation. . . . . . . . . . . . . . . . . . . 22

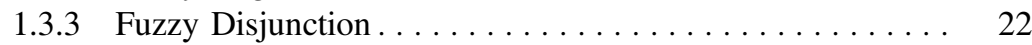

1.3.4 Fuzzy Implication . . . . . . . . . . . . . . . . . . 23

1.4 Linguistic Variables .......................... 25

1.5 Fuzzy Quantifiers .......................... 28

1.6 Some Remarks . . . . . . . . . . . . . . . . . . . . . . . . . . . 29

References............................ 30

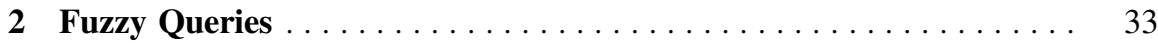

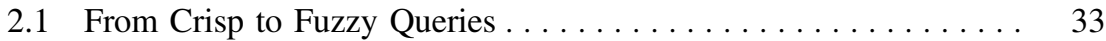

2.2 Construction of Fuzzy Sets for Flexible Conditions . . . . . . . . 37

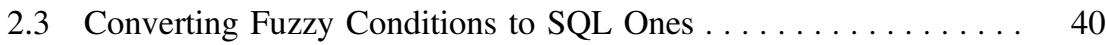

2.4 Calculation of Matching Degrees ................... 42

2.4.1 Independent Conditions Aggregated

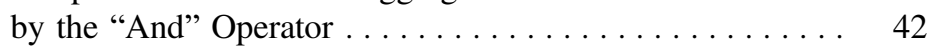

2.4.2 Fuzzy Preferences Among Atomic Query Conditions..... 44

2.4.3 Answer to the Second Atomic Condition Depends on the Answer to the First One. . . . . . . . . . . . . 47

2.4.4 Constraints and Wishes.................... 50

2.4.5 Quantified Queries $\ldots \ldots \ldots \ldots \ldots \ldots \ldots \ldots \ldots \ldots$ 
2.4.6 Querying Changes of Attributes over Time.......... 56

2.5 Empty and Overabundant Answers................ 57

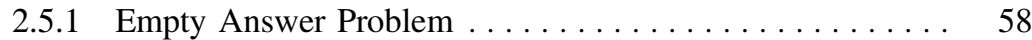

2.5.2 Overabundant Answer Problem................. 61

2.6 Some Issues Related to Practical Realization . . . . . . . . . . . . . 62

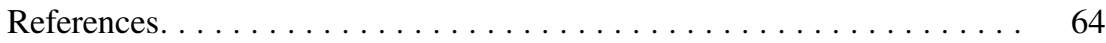

3 Linguistic Summaries ....................... 67

3.1 Benefits and Protoforms of Linguistic Summarization . . . . . . . 67

3.2 The Basic Structure of LS . . . . . . . . . . . . . . . . . . . . 69

3.3 Relative Quantifiers in Summaries . . . . . . . . . . . . . . . 70

3.4 LS with Restriction. . . . . . . . . . . . . . . . . . 73

3.5 Mining Linguistic Summaries of Interest . . . . . . . . . . . 75

3.6 Quality Measures of LSs . . . . . . . . . . . . . . . . . . 79

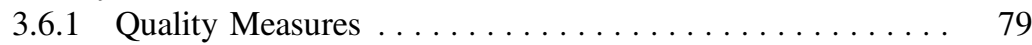

3.6.2 Aggregation of Quality Measures . . . . . . . . . . 83

3.6.3 Influence of Constructed Fuzzy Sets and T-Norms

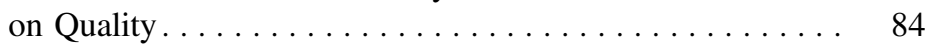

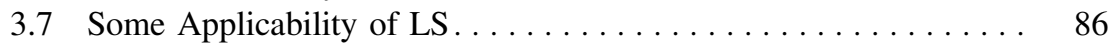

3.7.1 Quantified Queries (LS as a Nested Condition). . . . . . . 87

3.7.2 Generating IF-THEN Rules. . . . . . . . . . . . . . . 91

3.7.3 Estimation of Missing Values ............... 93

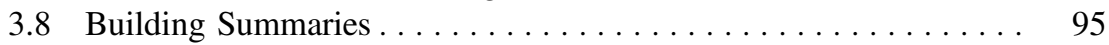

References. . . . . . . . . . . . . . . . . . . . . . . . 97

4 Fuzzy Inference . . . . . . . . . . . . . . . . . . . . . . 101

4.1 From Classical to Fuzzy Inference . . . . . . . . . . . . . . 101

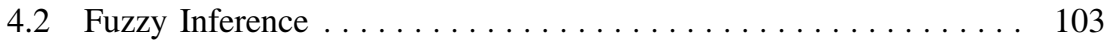

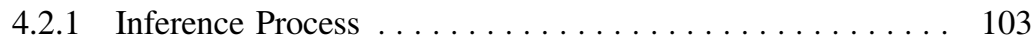

4.2 .2 Defuzzification $\ldots \ldots \ldots \ldots \ldots \ldots \ldots \ldots \ldots \ldots \ldots$

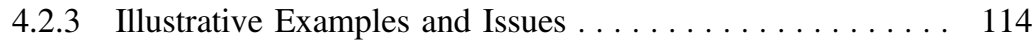

4.3 Fuzzy Inference Systems . . . . . . . . . . . . . . . . . . . 120

4.3.1 Mamdani Model (Logical Model) . . . . . . . . . . . . . . . 120

4.3.2 Sugeno Model (Functional Model) . . . . . . . . . . . . 122

4.4 Fuzzy Rule-Based System Design . . . . . . . . . . . . . . . . . . . . . . 123

4.5 Fuzzy Classification ........................ 126

4.5.1 A View on Crisp Classification. . . . . . . . . . . . . . 126

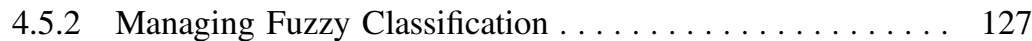

4.5.3 Fuzzy Classification by Fuzzy Queries . . . . . . . . . . . 130

4.6 Remarks to Applications. . . . . . . . . . . . . . . . . . . 133

References. . . . . . . . . . . . . . . . . . . . . . . 135

5 Fuzzy Data in Relational Databases. . . . . . . . . . . . . . . . 139

5.1 Classical Relational Databases . . . . . . . . . . . . . . . . . . 139

5.2 Fuzziness in the Data . . . . . . . . . . . . . . . . . . 141

5.3 Fuzzy Databases: An Overview . . . . . . . . . . . . . . . . . . . 142 
5.4 Basic Model of Fuzzy Database . . . . . . . . . . . . . . . . . . 143

5.4.1 Structure of Basic Model . . . . . . . . . . . . . . . . . . . 144

5.4 .2 Querying Basic Model .................. 145

5.5 Fuzzy Data in Traditional Relational Databases

Managed by Fuzzy Meta Model. . . . . . . . . . . . . . . . 147

5.5.1 Creating Fuzzy Meta Model . . . . . . . . . . . . . . . . . 148

5.5.2 Storing and Representing Tuples. . . . . . . . . . . . 151

5.5.3 Inserting Fuzziness into Existing Databases . . . . . . . . 155

5.5.4 Managing Fuzziness in Data and in Inference

Rules by the Same Database . . . . . . . . . . . . . . . 157

5.6 Querying Fuzzy Relational Databases. . . . . . . . . . . . . . . . 160

5.6.1 Aggregation Functions in Queries.............. 160

5.6 .2 Query Conditions ..................... 163

5.7 Linguistic Summaries on Fuzzy Data . . . . . . . . . . . . 171

5.8 Final Remarks . . . . . . . . . . . . . . . . . . . . . . . . 174

References. . . . . . . . . . . . . . . . . . . . 175

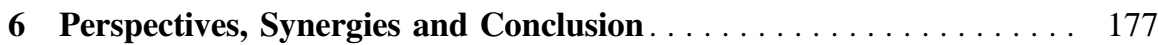

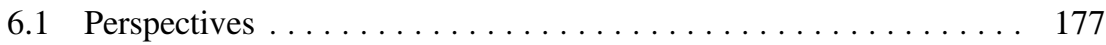

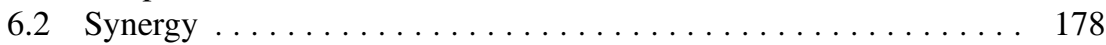

6.2.1 Fuzzy Inference and Fuzzy Databases. . . . . . . . . . 178

6.2.2 Linguistic Summaries and Fuzzy Inference. . . . . . . . . . 180

6.2.3 Fuzzy Queries for Fuzzy Classification and Summaries ... 181

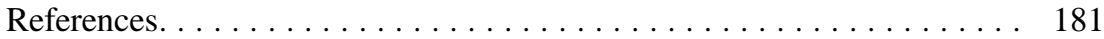

Appendix A: Illustrative Interfaces and Applications

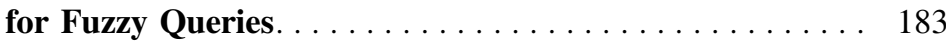

Appendix B: Illustrative Interfaces and Applications

for Linguistic Summaries. . . . . . . . . . . . . . . . 191

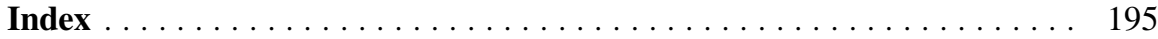




\section{Acronyms}

$\begin{array}{ll}\text { 1NF } & \text { First Normal Form } \\ \text { 2NF } & \text { Second Normal Form } \\ \text { 3NF } & \text { Third Normal Form } \\ \text { COG } & \text { Center of Gravity } \\ \text { COM } & \text { Center of Maxima } \\ \text { CRM } & \text { Customer Relationship Management } \\ \text { DBMS } & \text { Database Management System } \\ \text { DDL } & \text { Data Definition Language } \\ \text { DNF } & \text { Disjunctive Normal Form } \\ \text { FIS } & \text { Fuzzy Inference System } \\ \text { FMB } & \text { Fuzzy Meta-knowledge Base } \\ \text { FMM } & \text { Fuzzy Meta Model } \\ \text { GLC } & \text { Generalized Logical Condition } \\ \text { GMP } & \text { Generalized Modus Ponens } \\ \text { HDM } & \text { Height Defuzzification Method } \\ \text { HOF } & \text { Half of Field } \\ \text { IBA } & \text { Interpolative Realization of Boolean Algebra } \\ \text { LOM } & \text { Left of Maxima } \\ \text { LSs } & \text { Linguistic Summaries } \\ \text { MOM } & \text { Mean of Maxima } \\ \text { NFE } & \text { Necessarily Fuzzy Equal to } \\ \text { NFL } & \text { Necessarily Fuzzy Less than } \\ \text { OWA } & \text { Ordered Weighted Averaging Operator } \\ \text { PFE } & \text { Possibly Fuzzy Equal to } \\ \text { PFL } & \text { Possibly Fuzzy Less than } \\ \text { RDBMS } & \text { Relational Database Management System } \\ \text { ROM } & \text { Right of Maxima } \\ \text { SQL } & \text { Structured Query Language } \\ & \end{array}$




\section{Notations}

In each field variables, sets, properties, functions, etc. are either marked by letters using an (informal) agreement, or by letters the authors decided to use in their seminal papers. In this textbook we have decided to keep usual notation of most used terms and adjust the notation of other terms in order to avoid misinterpretation. As it is not always possible, some letters are not used for a single term only, but the explanation on their usage avoids misinterpretation of used letters and indexes.

\begin{tabular}{l|l}
\hline$A$ & Attribute, fuzzy set, answer to query \\
\hline $\bar{A}$ & Complement of fuzzy set \\
\hline$A^{(\alpha)}$ & $\alpha$-cut of fuzzy set \\
\hline$A_{c}$ & Accuracy \\
\hline$a, m, b$ & Parameters of fuzzy sets \\
\hline$B$ & Fuzzy set \\
\hline $\operatorname{core}(A)$ & Core of fuzzy set \\
\hline card(A) & Cardinality of fuzzy set, $|A|$ \\
\hline$c$ & Negation \\
\hline$c_{s}$ & Standard negation \\
\hline$c_{g}$ & Gödel negation \\
\hline$c_{d g}$ & Dual Gödel negation \\
\hline$C$ & Coverage \\
\hline$i_{C}$ & Coverage index \\
\hline$D$ & Domain, distance \\
\hline$d$ & Measure of fuzziness \\
\hline$E_{C}$ & Specificity \\
\hline$F$ & Fuzzification operator, fuzzy set \\
\hline$h$ & Height of fuzzy set \\
\hline$H$ & Highest value of attribute in a database \\
\hline$i_{S K D}$ & Kleene-Dienes implication \\
\hline & \\
\hline
\end{tabular}


(continued)

\begin{tabular}{|c|c|}
\hline$i_{s L}$ & Łukasiewicz implication \\
\hline$i_{q Z}$ & Zadeh implication \\
\hline$i_{r G d}$ & Gödel implication \\
\hline$i_{r G g}$ & Goguen implication \\
\hline$L$ & Lowest value of attribute in a database \\
\hline$N$ & Negative preference \\
\hline$O$ & Outlier measure \\
\hline$P$ & Predicate, positive preference \\
\hline$Q$ & Quantifier \\
\hline$Q_{c}$ & Quality of summary \\
\hline$r$ & Database tuple \\
\hline$R$ & Relation, rule, restriction \\
\hline$s$ & t-conorm or s-norm \\
\hline$s_{m}$ & Maximum s-norm \\
\hline$s_{a}$ & Algebraic sum \\
\hline$s_{L}$ & Łukasiewicz s-norm \\
\hline$s_{d}$ & Drastic s-norm \\
\hline $\operatorname{supp}(A)$ & Support of fuzzy set \\
\hline$S$ & Summarizer, simplicity \\
\hline$t$ & t-norm \\
\hline$t_{m}$ & Minimum t-norm \\
\hline$t_{p}$ & Product t-norm \\
\hline$t_{L}$ & Łukasiewicz t-norm \\
\hline$t_{d}$ & Drastic product \\
\hline$t_{n M}$ & Nilpotent minimum t-norm \\
\hline$T$ & Transformation \\
\hline$U$ & Usefulness \\
\hline$v$ & Validity of linguistic summary \\
\hline$w$ & Weight \\
\hline$X$ & Universe of disclosure, universal set \\
\hline$\alpha$ & And if possible operator \\
\hline$\beta$ & Or else operator, threshold \\
\hline$\delta$ & Firing degree of rule \\
\hline$\varepsilon$ & Length of slope of fuzzy set \\
\hline$\mu$ & Membership function \\
\hline$\varphi$ & Characteristic function \\
\hline$\theta$ & Length of flat segment of fuzzy set \\
\hline $\mathbb{N}$ & Set of natural numbers \\
\hline $\mathbb{R}$ & Set of real numbers \\
\hline
\end{tabular}

